\title{
Assessing Resolvability and Consistency in OBO Foundry Ontologies: Pilot Study
}

\author{
Shuxin $\mathrm{ZHANG}^{\mathrm{a}, 1}$, Nirupama BENIS ${ }^{\mathrm{a}}$ and Ronald CORNET ${ }^{\mathrm{a}}$ \\ ${ }^{a}$ Department of Medical Informatics, Amsterdam University Medical Center, \\ Amsterdam, The Netherlands
}

\begin{abstract}
Ontologies listed in the OBO Foundry are often regarded as reliable choices to be reused but ontology interoperability of them remains unknown. This study evaluated the resolvability of URIs and consistency of axioms in the OBO Foundry library, BFO ontology, and CIDO ontology. All had nonresolvable URIs, but the OBO library and the CIDO had additional interoperability issues regarding the use of incorrect prefixes, mixing up with ontologies, and inconsistency in the use of property. These detected issues reflected the real-world common problems that were not significant from human beings' point of view but hindered the machine-processability of ontologies. The assessment performed in this study was automated and enables scale-up against more metrics over more ontologies, which remains future work.
\end{abstract}

Keywords. Ontologies, Interoperability, Assessment, OBO Foundry

\section{Introduction}

Ontologies, as means to formalize concepts and relations that represent entities and their relations in a specific domain of the world, support health data in being Findable Accessible, Interoperable, and Reusable (i.e., FAIR). Given a large number of ontologies developed [8][7], ontology interoperability is needed to prevent misunderstanding and isolation between different resources [1]. The Open Biological and Biomedical Ontologies (OBO) Foundry [8] is an ontology library making registered ontologies more findable and reusable. These ontologies are reviewed and listed in the OBO Foundry library, and in practice, they are often regarded as "good" choice to be reused. However, "being commonly-used" or "being of good reputation"2 are not the golden standards but subjective preferences, which can make people unconsciously unaware of detectable pitfalls that should not be further spread over by those "good" ontologies. In this pilot study, we examined the resolvability of URIs and consistency of RDF triples of published ontologies in the $\mathrm{OBO}$ Foundry library to explore the real-world interoperability issues.

\footnotetext{
${ }^{1}$ Corresponding Author.

$2 \mathrm{http} / /$ purl.org/ist/interoperability-key-point\#commonly-used-vocabulary
} 


\section{Methods}

We applied the assessment approach from [10] which evaluates the interoperability of existing linked dataset in RDF. Five metrics (see Table 1) were selected because:

- they are objective with minimal human involvement;

- their testing can be automated;

- they reflect four different dimensions ${ }^{3}$.

Table 1. List of metrics implemented in this study and their reflected dimensions.

\begin{tabular}{cll}
\hline \multicolumn{1}{c}{ Dimension } & \multicolumn{1}{c}{ Metric } & \multicolumn{1}{c}{ Interpretation } \\
\hline Availability & Resolvability of URIs & $\begin{array}{l}\text { Upon request of a URI term, check whether any } \\
\text { information is provided as result. }\end{array}$ \\
$\begin{array}{c}\text { Representational- } \\
\text { consistency }\end{array}$ & Reuse of existing terms & Detect the use of existing terms. \\
Understandability & Use of Human-readable Labelling & $\begin{array}{l}\text { Detect the use of human-readable annotations. } \\
\text { If classes are correctly used as objects in rdf:type triples. } \\
\text { If properties are correctly used as predicates in triples. }\end{array}$ \\
Consistency & $\begin{array}{l}\text { Misused Properties of the } \\
\text { Iype owl:DatatypeProperty } \\
\text { or owl:ObjectProperty }\end{array}$ & $\begin{array}{l}\text { If objects of properties as type owl:ObjectProperty are } \\
\text { are literal. }\end{array}$ \\
\hline
\end{tabular}

Three RDF datasets were evaluated (see Table 2) as representatives of their types. BFO and CIDO, which are represented in the Web Ontology Language (OWL) ${ }^{4}$, are regarded as RDF datasets in this study. BFO has been manually reviewed by experts in the OBO Foundry community while reviewing CIDO is not completed yet. They are commonlyused and can serve as the starting point for assessing ontologies in OBO Foundry.

Table 2. List of evaluated RDF datasets.

\begin{tabular}{lll}
\hline Dataset & Type & Description \\
\hline OBO Library & Metadataset & $\begin{array}{l}\text { A dataset which lists current OBO ontologies with their meta information, } \\
\text { including activity status, access URI, theme, and etc. } \\
\text { An upper-level ontology in support of domain ontologies developed for } \\
\text { scientific research within the framework of OBO Foundry. }\end{array}$ \\
CIDO & $\begin{array}{l}\text { Upper Ontology } \\
\text { Ontology }\end{array}$ & A biomedical ontology in the area of coronavirus infectious disease. \\
\hline
\end{tabular}

Unique URIs were extracted from RDF datasets to check resolvability, which means that an HTTP request for these URIs can provides us with other resources. Results were described by HTTP status code. Diagram of workflow can be found at a persistent URL ${ }^{5}$.

RDF triples were extracted and then divided into triples with or without the property rdf:type. The types of objects in rdf:type triples were checked if they were of type owl:Class. Predicates in other triples were checked if they were of any property type (e.g., rdfs:Property). After that, we examined if these classes and properties were processable by machines, which checks 1) if any content can be automatically retrieved via either parsing through a parser or querying through a SPARQL wrapper and 2) if retrieved content contains given resource of that URI. For the predicates whose types

\footnotetext{
${ }^{3}$ https://purl.org/iqd

${ }^{4}$ https://www.w3.org/TR/owl2-overview/

${ }^{5}$ https://purl.org//report/workflow
} 
were specified as either owl:DatatypeProperty (whose objects should be literal) or owl:ObjectProperty (whose objects should be URI), their objects in triples were checked. Any URI, class, predicate, or triple that was assessed but failed against metrics was regarded as a failure case, which served as the unit for analysis. We utilized the parser and SPARQL wrapper developed in $r d f l i b^{6}$ package. The SPARQL endpoints were Ontobee $^{7}$ and BioPortal ${ }^{8}$. Implementation scripts can be found at GitHub ${ }^{9}$.

\section{Results}

Table 3 describes the number of failure cases detected in the OBO library dataset, BFO and CIDO against metrics. The OBO library dataset has failures in unavailability of 38 (out of 1,067) URIs, unretrievability of 5 (out of 23) predicates, and 8 (out of 14) misused properties of owl:ObjectProperty, and these failures also occurred in CIDO, while the BFO dataset has failures only in unavailability of 70 URIs (out of 156). In terms of Understandability, all datasets applied human-readable labelling, including rdfs:label and det:description.

All test datasets have problems in resolvability of URIs and some of them stemmed from the same resource. In the OBO library dataset, all terms with the prefix "http://obofoundry.github.io/vocabulary/" were not found (HTTP 404). Some URIs are those referring to deprecated ontologies, e.g., <http://purl.obolibrary.org/obo/epo.owl $>$ In the BFO, all URIs with the prefix "http://purl.obolibrary.org/obo/bfo/axiom/" are not found (HTTP 404), which amount to 67 (out of 70). In the OBO dataset, a property $<$ http://purl.org/dc/terms/1.1/theme $>$ was used but it does not exist, though that URI is still resolvable to DCMI Metadata Terms (DCT) ontology. Through query via a SPARQL endpoint, this error was detected as that query was performed by extract pattern matching. $<$ http://www.w3.org/ns/dcat\#theme $>$ from Data Catalog Vocabulary (DCAT), however, exists. So it is important to distinguish terms between DCT and DCAT, alike but different. Besides, DCT maintains two namespaces: "http://purl.org/dc/elements/1.1/" and "http://purl.org/dc/terms/" but not "http://purl.org/dc/terms/1.1/". So the URI <http://purl.org/dc/terms/1.1/license $>$ in the OBO library dataset could not resolve to any content regarding license and was not queriable in SPARQL endpoint. Therefore, we should be aware of correct use of DCT namespaces, though such mistake still can guide you towards DCT resources but it is not processable by machines. Eight failed properties of owl:ObjectProperty in the OBO dataset and ten failure in CIDO are listed ${ }^{10}$ with number of involved RDF triples. In all of these failed triples, we found that all objects were the string version of an URI instead of the Notation 3 format $^{11}$. Below is an example:

\footnotetext{
<http://purl.obolibrary.org/obo/obi>

<http://usefulinc.com/ns/doap\#bug-database>

"http://purl.obolibrary.org/obo/obi/tracker"
}

\footnotetext{
${ }^{6}$ https://github.com/RDFLib/rdflib

7 http://www.ontobee.org/sparql

${ }^{8}$ http://sparql.bioontology.org/

${ }^{9}$ https://github.com/sxzhang1201/Interoperable-Supportive-Tool

$10 \mathrm{https}$ ://purl.org/obo_library_assess/object_property

11 https://www.w3.org/TeamSubmission/n3/
} 
Table 3. Number of failure cases against metrics for OBO library and BFO.

\begin{tabular}{|c|c|c|c|c|c|c|}
\hline \multirow{2}{*}{ Metrics } & \multicolumn{2}{|c|}{ OBO Library Dataset } & \multicolumn{2}{|c|}{$\mathrm{BFO}$} & \multicolumn{2}{|c|}{ CIDO } \\
\hline & \# Total & \# Failure (\%) & \# Total & \# Failure (\%) & \# Total & \# Failure (\%) \\
\hline \multicolumn{7}{|l|}{ Resolvability of URIs } \\
\hline - Available URIs & 1067 & $38(4 \%)$ & 156 & $70(45 \%)$ & 9598 & $2183(23 \%)$ \\
\hline \multicolumn{7}{|l|}{ Reuse of existing terms } \\
\hline $\begin{array}{l}\text { - URI of a class resolving to } \\
\text { content concerning that class }\end{array}$ & 4 & 0 & 19 & 0 & 2249 & $117(5 \%)$ \\
\hline $\begin{array}{l}\text { - URI of a predicate resolving } \\
\text { to content concerning that } \\
\text { predicate }\end{array}$ & 23 & $5(22 \%)$ & 26 & 0 & 164 & $33(20 \%)$ \\
\hline \multicolumn{7}{|l|}{$\begin{array}{l}\text { Misplaced classes or } \\
\text { properties }\end{array}$} \\
\hline $\begin{array}{l}\text { - Classes incorrectly used as } \\
\text { properties }\end{array}$ & 4 & 0 & 19 & 0 & 2132 & 0 \\
\hline $\begin{array}{l}\text { - Properties incorrectly used as } \\
\text { classes }\end{array}$ & 23 & 0 & 26 & 0 & 131 & 0 \\
\hline \multicolumn{7}{|l|}{$\begin{array}{l}\text { Misused Properties of the } \\
\text { type owl:DatatypeProperty } \\
\text { or owl:ObjectProperty }\end{array}$} \\
\hline $\begin{array}{l}\text { - Properties of } \\
\text { owl:DatatypetProperty }\end{array}$ & 1 & 0 & 5 & 0 & 40 & $4(10 \%)$ \\
\hline $\begin{array}{l}\text { - Properties of } \\
\text { owl:ObjectProperty }\end{array}$ & 14 & $8(57 \%)$ & 4 & 0 & 13 & $10(77 \%)$ \\
\hline \multicolumn{7}{|l|}{ Human-readable Labelling } \\
\hline - Human-readable annotations & 609 & 0 & 50 & 0 & 9402 & 0 \\
\hline
\end{tabular}

\section{Discussion}

In this study, we found that well-used ontologies from a reliable platform contained errors, including non-resolvability of URIs, use of incorrect prefixes, mixing up with ontologies, and inconsistency in the use of property. Both OBO library and BFO maintain their own vocabularies, all of which, however, are not resolvable. It is probably ascribed to authorization issues and further information is needed by reaching out to their authors. URIs referring to deprecated ontologies were not but should be resolvable along with version information so that those still using outdated ones are able to find related update activity and reach out to the updated ones. Many researchers have performed quality assessment of ontologies. Burton-Jones et al.[2] proposed a suite of metrics, i.e., Syntactic, Semantic, Pragmatic, and Social, to evaluate the usefulness of ontologies found in the DARPA Agent Markup Language (DAML) library. The only metric relevant to Consistency in [2] measures the proportion of inconsistent classes and properties but does not clarify how such inconsistency could be detected. Duque-Ramos et al.[4] adapted a Software Engineering standard, Software product Quality Requirements and Evaluation (SQuaRE) to develop a framework for ontology evaluation. Fourteen metrics were defined to assess the quality of ten ontologies of "units of measurements" and "cell types". These metrics were measured in an automated manner but focused on "demographics", for example, measuring the number of attributes per class, and the mean number of direct subclasses. He et al.[5] proposed an "eXtensible Ontology Development" strategy and four associated principles (i.e., ontology reuse, ontology semantic alignment) to provide high-level guideline for ontology development. Our study instead focused on a relatively lower level of quality assessment enabling 
resolvability and consistency checking in an automated and expectedly complete fashion.Our study employed an automated approach to assess a set of metrics reflecting different dimensions of ontology interoperability. Such automation enables to evaluate more datasets in an objective way. However, there are more quality metrics in [10] that are not tested. The tool implemented is capable of a limited number of metrics but incorporation with other existing tools, e.g., Luzzu[3] and RDFUnit[6], can support the expansion of quality assessment. An integrated assessment approach performed by Sanju et al.[9] is also promising to detect additional interoperability problems but inconsistency of performance among different assessment tools should be addressed. True machine readability of ontologies, concepts and classes is key to supporting reasoning over data and establishing FAIR linkable data. Consequently, the quality of such ontologies should be maximal, hence quality assessment should be applied, and should be facilitated. Our approach contributes to quality assessment, and the developed tool automates such assessment. In the future, with more metrics incorporated, more ontologies should be assessed to capture a comprehensive view of common interoperability problems in existing well-used ontologies of a specific domain, for example, ontologies concerning COVID-19.

\section{Conclusions}

Even established, well-used ontologies aren't free of errors that can be automatically detected. We have developed tooling that helps to detect and resolve errors. Further work and research are needed to detect more types of errors over more ontologies.

\section{References}

[1] Amith M, He Z, Bian J, Lossio-Ventura JA, Tao C. Assessing the practice of biomedical ontology evaluation: Gaps and opportunities. Journal of biomedical informatics. 2018 Apr 1;80:1-3.

[2] Burton-Jones A, Storey VC, Sugumaran V, Ahluwalia P. A semiotic metrics suite for assessing the quality of ontologies. Data \& Knowledge Engineering. 2005 Oct 1;55(1):84-102.

[3] Debattista J, Auer S, Lange C. Luzzu - a methodology and framework for linked data quality assessment. Journal of Data and Information Quality (JDIQ). 2016 Oct 25;8(1):1-32..

[4] Duque-Ramos A, et al. OQuaRE: A SQuaRE-based approach for evaluating the quality of ontologies. Journal of research and practice in information technology. 2011 May;43(2):159-76.

[5] He Y, et al. The eXtensible ontology development (XOD) principles and tool implementation to support ontology interoperability. Journal of biomedical semantics. 2018 Dec;9(1):1-0.

[6] Kontokostas D, Westphal P, et al. Test-driven evaluation of linked data quality. InProceedings of the 23rd international conference on World Wide Web 2014 Apr 7 (pp. 747-758)..

[7] Corbin-Lickfett KA, et al. The HSV-1 ICP27 RGG box specifically binds flexible, GC-rich sequences but not G-quartet structures. Nucleic acids research. 2009 Nov 1;37(21):7290-301..

[8] Smith B, Ashburner M, et al. The OBO Foundry: coordinated evolution of ontologies to support biomedical data integration. Nature biotechnology. 2007 Nov;25(11):1251-5.

[9] Tiwari S, Abraham A. Semantic assessment of smart healthcare ontology. International Journal of Web Information Systems. 2020 Jul 31.

[10] Zhang S, Benis N, De Keizer N, Cornet R. An Approach for Interoperability Assessment of RDF Data, Submitt. to Semant. Web J. (2021). 